\title{
Epilepsia y embarazo. Un estudio comparativo dos décadas después
}

\author{
Paola Barriguete-Chávez Peón ${ }^{1}$, Blanca Eugenia Farfán-Labonne1, Jorge Miguel Ibarra-Puig1, \\ Efraín Olivas-Peña ${ }^{1}$, Mónica Flores-Ramos², Daniela Chinchilla-Ochoa ${ }^{1}$ y Saúl Garza-Morales ${ }^{1}$ \\ ${ }^{1}$ Departamento de Neurociencia, Instituto Nacional de Perinatología Isidro Espinosa de los Reyes; ${ }^{2}$ Instituto Nacional de Psiquiatría Ramón de la \\ Fuente Muñiz. Ciudad de México, México
}

\begin{abstract}
Resumen
Antecedentes: La epilepsia es la enfermedad neurológica más frecuente durante el embarazo. Los fármacos antiepilépticos (FAE) de primera generación implican un efecto teratogénico directo y mayor probabilidad de complicaciones madre-hijo. Durante las últimas tres décadas, las modificaciones epidemiológicas en la presentación de la epilepsia han condicionado los cambios en Ios tratamientos farmacológicos preferidos. Estos cambios afectan a los resultados perinatales en mujeres embarazadas con esta condición; sin embargo, no hay estudios comparativos de esta naturaleza. Objetivos: Comparar las preferencias del FAE y el resultado perinatal durante las últimas décadas en la población mexicana. Métodos: Mujeres epilépticas embarazadas $(n=275)$ y sus hijos recibieron valoración y seguimiento clínico de médicos especialistas (obstetras, neurólogos, genetistas), y se compararon con un estudio de 100 mujeres epilépticas embarazadas y sus hijos estudiados en 1996. Todos los pacientes fueron estudiados en el Instituto Nacional de Perinatología (INPer) (Ciudad de México). Resultados: La administración de monoterapia incrementó del 61 al 73\% y la frecuencia de uso de ácido valpróico (AVP) aumentó del 8 al $45 \%$. Las malformaciones fueron más frecuentes en el estudio actual, aumentando las dismorfias menores del 8 al 15\% y las dismorfias mayores del 0 al $4 \%$. Conclusiones: La tendencia del uso de AVP en mujeres mexicanas debe ser explorada como posibles variables involucradas en el resultado perinatal. Deben considerarse tratamientos alternativos con tasas teratogénicas más bajas para las mujeres fértiles.
\end{abstract}

Palabras clave: Fármaco antiepiléptico. Resultado perinatal. Dismorfias. Embarazo. Enfermedad neurológica.

\section{Epilepsy and pregnancy. A comparative study two decades afterwards}

\section{Abstract}

Background: Epilepsy is the most frequent neurologic disease during pregnancy. First generation antiepileptic drugs involve a direct teratogenic effect and imply an increased probability of mother-child complications. During the last three decades, epidemiological modifications in epilepsy presentation have conditioned changes in the pharmacological treatments preferred.

\section{Correspondencia:}

Paola Barriguete-Chávez Peón

Departamento de Neurociencia

Instituto Nacional de Perinatología Isidro

Espinosa de los Reyes

Montes Urales, 825

Lomas Virreyes, Del. Miguel Hidalgo,

C.P. 14000,Ciudad de México, México

Fecha de recepción: 08/01/2018

Fecha de aceptación: 15/05/2018

E-mail: paolabarriguete@gmail.com
Disponible en internet: 13-11-2018 Rev Mex Neuroci. 2018;19(5):27-36 www.revmexneuroci.com 2018. Academia Mexicana de Neurologia A.C. Publicado por Permanyer México. Este es un articulo Open Access bajo la licencia CC BY-NC-ND 
These changes impacts perinatal outcomes in pregnant women with this condition, however there are no comparative studies of this nature. Objectives: To compare antiepileptic drug (AED) preferences and perinatal outcome during the last decades in the Mexican population. Methods: Pregnant epileptic women $(n=275)$ and their children, received clinical assessment and follow-up from medical specialists (obstetrician, neurologist, geneticist), and compared with a study of 100 pregnant epileptic women and their children studied in 1996. All patients were studied at the National Institute of Perinatology (INPer), in Mexico City. Results: The administration of monotherapy increased from 61 to $73 \%$, and the frequency of valproate use raised (8-45\%). Malformations were more frequent in the current study, increasing minor dysmorphias from 8 to $15 \%$ and mayor dysmorphias from 0 to $4 \%$. Conclusions: Mexican women's tendency to use valproate should be explored as possible variables involved in perinatal outcome. Alternative treatments with lower teratogenic rates should be considered for fertile women.

Key words: Neurologic Disease. Antiepileptic Drug. Perinatal Outcome. Dysmorphias. Pregnancy.

\section{Introducción}

La epilepsia es la enfermedad neurológica más común que se presenta durante el embarazo, con una prevalencia internacional del 0.2 al $0.5 \%{ }^{1,2}$. En el INPer de Ciudad de México, se describe una frecuencia del $1.6 \%{ }^{3}$.

Este trastorno y el uso de los antiepilépticos altera el desarrollo del embarazo a través de diversos mecanismos: complica la frecuencia de las crisis $^{4}$, reduce los accidentes ${ }^{5}$ y aumenta el riesgo de malformación fetal ${ }^{6,7}$ y las complicaciones perinatales ${ }^{8}$.

En la última década, la Food and Drug Administration (FDA) ha autorizado al menos cinco nuevos antiepilépticos para uso clínico, pero se necesitan más pruebas acerca de su seguridad, eficacia y potencial teratogénico durante el embarazo $0^{9}$. La investigación define la oxcarbacepina (Ox-CBZ), el levetiracetam (LVT) y la lamotrigina (LMT) como los fármacos con más información de seguridad y menor riesgo de malformación, mientras que la gabapentina (GPT) y el topiramato (TOP) tienen datos insuficientes para apoyar la falta de riesgo $0^{6,9}$.

Los antiepilépticos tradicionales -carbamacepina (CBZ), fenobarbital (FB) y fenitoína (DFH)- muestran una tasa dismórfica similar a los nuevos antiepilépticos, en contraste con el ácido valproico (AVP), que aumenta al doble el riesgo de malformación, especialmente los defectos del tubo neural $1^{6,10}$.

La teratogenicidad potencial de los FAE durante el embarazo es muy conocida, y se relaciona principalmente con una tasa dismórfica dependiente de unas dosis de $700 \mathrm{mg}$ diarios de AVP, $400 \mathrm{mg}$ diarios de CBZ y $200 \mathrm{mg}$ diarios de LMT, respectivamente ${ }^{6,11}$.

México tiene reportes insuficientes de casos referidos a la evolución del embarazo en mujeres epilépticas $^{12-14}$, así como de sus efectos neonatales. No existe un resultado comparativo de la evolución del uso de los antiepilépticos y los resultados perinatales.
El objetivo principal de nuestro trabajo es comparar los avances clínicos, las preferencias antiepilépticas y los resultados perinatales en una población actual y otra analizada hace $20 \mathrm{años}^{13}$. Ambos estudios fueron planeados por el mismo grupo de investigadores en los hospitales de tercer nivel del Instituto Nacional de Salud Pública, localizado en Ciudad de México.

\section{Materiales y métodos}

Se realizó un análisis retrospectivo basado en la población de mujeres epilépticas con embarazos simples que resolvieron su embarazo en el INPer de enero de 2008 a junio de 2014, utilizando la base de datos perinatal del hospital. A su vez, se comparó con un estudio realizado en 1996 que analizó la misma base de datos de agosto de 1991 a febrero de 1993.

Los criterios para participar en la evaluación retrospectiva fueron el diagnóstico de epilepsia (según International League Against Epilepsy (ILAE) ${ }^{15}$ ) y el embarazo (confirmado con pruebas inmunológicas). El estudio incluyó pacientes que usaron el FAE (con una ingesta regular 0 irregular), otras que nunca lo usaron y otras que lo suspendieron después de saber que estaban embarazadas. Las mujeres con enfermedades psiquiátricas, discapacidad intelectual y dependencia de drogas fueron excluidas del estudio.

Todas las pacientes recibieron un servicio clínico neurológico, suplementos de ácido fólico (1 $\mathrm{mg}$ diario, al menos desde la primera visita clínica al INPer $)^{15-17}$. Asimismo, se recopilaron datos acerca de los análisis electroencefalográficos (EEG), de los ultrasonidos fetales y de la vigilancia obstétrica especializada realizada en cada una de ellas. El Servicio Clínico Neurológico incluyó una visita mensual con una dosis y un ajuste del FAE, según las crisis epilépticos y la información del EEG.

Según la reglamentación oficial mexicana, los recién nacidos recibieron un servicio neonatal especializado 
que incluía: evaluación física, neurológica y de la edad gestacional, así como profilaxis con vitamina $\mathrm{K}$, entre otros aspectos ${ }^{16,17}$. Se tomaron en cuenta los estudios de imagen (tomografía o resonancia magnética) realizados antes del embarazo o aquéllos que se llevaron a cabo posteriormente en emergencias médicas, como convulsiones no controladas o alguna sintomatología clínica que sugiriera una lesión cerebral ocupativa.

La información extraída de la base de datos perinatal se clasificó y archivó en las siguientes categorías:

- Características maternas: edad, paridad, diagnóstico, inicio de la epilepsia, convulsiones durante el embarazo y otros diagnósticos.

- Tratamiento: tipo, dosis, monoterapia o politerapia, inicio del trimestre y cese del tratamiento.

- Complicaciones maternas perinatales: trastornos hipertensivos inducidos por el embarazo tales cómo, hidramnios, oligohidramnios, restricción del crecimiento intrauterino y placenta previa.

- Resolución del embarazo: tipo de resolución, indicaciones de cesárea o abortos.

- Resultados perinatales: malformaciones congénitas, líquido amniótico teñido de meconio, patrones de frecuencia cardíaca fetal inestables, peso al nacer, puntuaciones de Apgar y mortalidad perinatal.

La evolución obstétrica, el tratamiento farmacológico, la frecuencia y las causas de las crisis, la resolución del embarazo, la somatometría y la evaluación neonatal (clínica y hospitalaria) se registraron y compararon con los resultados descritos hace 20 años $^{13}$. Nuestro principal objetivo fue evaluar la prevalencia de malformaciones congénitas importantes después del nacimiento en los hijos expuestos a monoterapia y politerapia (AVP) antiepiléptica, divididos en intervalos de rango de dosis. Los médicos, clasificados por un comité independiente, que definieron malformaciones congénitas mayores y menores, ordenadas según los criterios de Supervisión Europea de Anomalías Congénitas (EUROCAT), registraron anormalidades en el recién nacido de forma detallada ${ }^{18}$.

El análisis de frecuencia y las estadísticas descriptivas se llevaron a cabo utilizando el SPSS 12.0.

\section{Resultados}

De las 290 mujeres identificadas a posteriori con un diagnóstico confirmado de epilepsia, se excluyeron 15 casos (10 de ellos por alguna inconsistencia en el diagnóstico y 5 porque los datos estaban incompletos). El análisis incluyó a 275 mujeres, con un promedio de 25 años \pm 6.17 (en un rango de 13 y 43 años). La edad promedio de inicio de los síntomas epilépticos fue a los 14 años \pm 7.04 (en un rango de 0 a 36 años), de los cuales el 83.8\% comenzó la sintomatología antes de los 20 años.

El tipo de crisis epiléptica más frecuente fue la tonicoclónica motora de comienzo generalizado, mostrada en 153 pacientes (46.9\%), seguida por la de ausencias no motoras, también de inicio generalizado, en 41 pacientes $(12.5 \%)$, y por la tonicoclónica focal a bilateral en 31 casos (11.3\%) (Tabla 1).

El tratamiento farmacológico más utilizado para controlar las crisis fue la monoterapia, administrada a 204 mujeres $(74.2 \%)$, seguido por la politerapia, administrada a $48(17.5 \%)$, mientras que otras 23 pacientes ( $8.4 \%)$ no recibieron tratamiento. En la monoterapia, los FAE, más utilizados fueron de AVP (46\%), OXC (45.8\%) y LMT (3.5\%). En la politerapia se utilizó principalmente AVP/CBZ (37.2\%) (Tabla 2).

Se administró una dosis baja de AVP $(<700)$ al $73 \%$ de las pacientes, una dosis media $(>700-1,500)$ al $22.3 \%$ y una dosis alta $(>1,500)$ al $4.4 \%$. Asimismo, se administró una dosis baja de CBZ (< 400) al 70\% de las pacientes.

Durante el primer trimestre, un $90.1 \%$ de las mujeres embarazadas recibió tratamiento médico, cifra que se incrementó al $92.7 \%$ en el segundo trimestre y al $93.4 \%$ en el tercero.

Un $68 \%$ de las pacientes tuvieron crisis epilépticas durante el embarazo, con un promedio de res crisis durante todo el embarazo. La interrupción del tratamiento (15.8\%), los bajos niveles de FAE $(7 \%)$ y el uso de un tratamiento genérico $(2.2 \%)$ fueron las principales causas de las convulsiones no controladas.

En 182 pacientes (66.2\%) se observaron ondas paroxísticas anormales sugestivas de epilepsia (onda aguda focal o generalizada y/o complejos de punta-onda). Asimismo, se identificaron complicaciones perinatales en 128 mujeres (46.5\%), entre las que predominaron la preeclampsia (25 casos [19.5\%]), el riesgo del bienestar fetal (11.7\%) y el virus del papiloma humano $(7.3 \%)$.

La resolución del embarazo se realizó por cesárea en el $74.5 \%$ de las pacientes y por parto vaginal en el $24 \%$ (en 18 de 86 pacientes se utilizaron fórceps). Además, se registraron abortos en tres mujeres (1.5\%). Las indicaciones más frecuentes de cesárea fueron: desproporción cefalopélvica (15.7\%), falta de progresión del parto $(13.5 \%)$ y preeclampsia $(9.7 \%)$.

Entre las pacientes embarazadas nacieron 134 recién nacidas, que presentaron los siguientes datos somatométricos: peso de 2,936 $\pm 434 \mathrm{~g}$ (en un rango de 1,010 a 3,982 ), altura de $48.5 \pm 2.6 \mathrm{~cm}$ (en un rango 
Rev Mex Neuroci. 2018;19

Tabla 1. Tipo de crisis epiléptica

\begin{tabular}{|c|c|c|c|c|c|}
\hline \multicolumn{3}{|l|}{ Estudio de 1996} & \multicolumn{3}{|l|}{ Estudio de 2017} \\
\hline Tipo de crisis & $n=100$ & $\%$ & Tipo de crisis & $n=275$ & $\%$ \\
\hline $\begin{array}{l}\text { Tonicoclónico motor de comienzo } \\
\text { generalizado }\end{array}$ & 54 & 34.83 & $\begin{array}{l}\text { Tonicoclónico motor de comienzo } \\
\text { generalizado }\end{array}$ & 153 & 46.9 \\
\hline \multirow[t]{2}{*}{ Tonicoclónico focal a bilateral } & 31 & 20 & Tonicoclónico focal a bilateral & 41 & 12.57 \\
\hline & & & $\begin{array}{l}\text { Conciencia focal deteriorada de inicio } \\
\text { no motor }\end{array}$ & 35 & 10.73 \\
\hline $\begin{array}{l}\text { Conciencia focal deteriorada de inicio no } \\
\text { motor }\end{array}$ & 25 & 16.12 & Conciencia focal de inicio no motor & 31 & 9.50 \\
\hline Conciencia focal de inicio no motor & 18 & 11.61 & Generalizado (otro motor: mioclónico) & 0 & 0 \\
\hline Generalizado (otro motor: mioclónico) & 14 & 9.03 & $\begin{array}{l}\text { Ausencias no motoras de inicio } \\
\text { generalizado }\end{array}$ & 26 & 7.97 \\
\hline $\begin{array}{l}\text { Ausencias no motoras de inicio } \\
\text { generalizado }\end{array}$ & 7 & 4.51 & $\begin{array}{l}\text { Conciencia focal deteriorada de inicio } \\
\text { no motor }\end{array}$ & 25 & 7.66 \\
\hline $\begin{array}{l}\text { Conciencia focal deteriorada de inicio } \\
\text { motor }\end{array}$ & 5 & 3.22 & Generalizado (otro motor: tónico) & 15 & 4.60 \\
\hline Generalizado (otro motor: tónico) & 1 & 0.58 & & & \\
\hline
\end{tabular}

El número y el porcentaje de pacientes con cada tipo de ataque epiléptico se presentan de acuerdo a los estudios de 1996 y 2017 . Los pacientes presentaron más de un tipo clínico.

de 36.5 a 53) y circunferencia de la cabeza (CDC) de $33.5 \pm 2.02 \mathrm{~cm}$ (en un rango de 17.7 a 37 ). Los datos de los varones recién nacidos (138) fueron los siguientes: peso de 2,880 $\pm 549.8 \mathrm{~g}$ (en un rango de 1,355 a 4,305 ), altura de $48 \pm 2.6 \mathrm{~cm}$ (en un rango de 32 a 54 ) y CDC de $33.6 \pm 1.6 \mathrm{~cm}$ (en un rango de 28 a 39 ). El $86.3 \%$ de todos los descendientes se caracterizaron como eutróficos y el $87.4 \%$ dentro del término (Tabla 3 ).

La puntuación Apgar fue $\leq$ a 5 en 15 casos (5.5\%) y $<8$ en 7 casos a los $5 \mathrm{~min}$ (1.5\%). Un total de 11 lactantes (4\%) presentaron sufrimiento fetal. Con la evaluación de Silverman, 252 niños tenían dificultad respiratoria leve (92.6\%), 8 (2.9\%) mostraron dificultad respiratoria moderada y $2(0.8 \%)$ dificultad respiratoria grave. En total, 53 neonatos (19.3\%) requirieron reanimación.

Se identificaron dismorfismos menores en 32 casos (12\%) y 7 casos con dismorfia mayor (3\%), con 2 casos de defectos del tubo neural $(0.72 \%)$, ambos expuestos a FAE (uno de ellos expuesto a 1,200 mg diarios de AVP y $225 \mathrm{mg}$ diarios de LTG, y el otro a $600 \mathrm{mg}$ diarios de CBZ) (Tabla 4).

\section{Resultados comparativos}

La edad promedio de las pacientes al ingresar en el instituto fue de 25 años (en ambos estudios iban de los 13 a los 43 años). El inicio de la epilepsia antes de los 20 años de edad ocurrió en el $80 \%$ de los casos notificados en 1996, mientras que en el estudio actual fue en el 47\%. En la investigación de 1996 predominó la epilepsia de inicio focal ( $n=79$ pacientes), en contraste con la actual, en la que se observó un mayor número de convulsiones de inicio generalizado en todas sus variedades (Tabla 1).

El porcentaje de pacientes que recibieron monoterapia aumentó del 61 al 73\%, mientras que las mujeres que no recibieron medicamentos antiepilépticos durante el embarazo se redujeron a la mitad (del 18 al 9\%). La frecuencia de uso de AVP aumentó cinco veces entre el estudio de 1996 y el actual (del 8 al 45\%), reduciendo el uso de la DFH (Tabla 2).

La suspensión voluntaria de los FAE fue el factor más común asociado con las convulsiones no controladas. En 1996 el porcentaje fue del 50\%, y en 2017, del $24 \%$.

En el estudio de 1996, de las complicaciones perinatales, el 24\% $(n=9)$ se debió a infecciones urinarias, el $14 \%(n=5)$ a cervicovaginitis y otro $14 \%(n=5)$ a hipertensión gestacional aguda. De las observadas en 2017 , el $20 \%(n=25)$ fueron por preeclampsia, el $12 \%$ $(n=15)$ por riesgo de bienestar fetal y un $7 \%(n=9)$ por el virus del papiloma humano. Ambos estudios compartieron complicaciones perinatales similares. 
Tabla 2. Farmacoterapia antiepiléptica

\begin{tabular}{|c|c|c|c|c|c|}
\hline \multicolumn{3}{|l|}{ Estudio de 1996} & \multicolumn{3}{|l|}{ Estudio de 2017} \\
\hline Tratamiento farmacológico & $n=100$ & $\%$ & Tratamiento farmacológico & $\mathrm{n}=\mathbf{2 7 5}$ & $\%$ \\
\hline Monoterapia & 61 & 61 & Monoterapia & 204 & 74.18 \\
\hline DFH & 27 & 44.26 & AVP & 92 & 45.77 \\
\hline CBZ & 24 & 39.34 & CBZ & 79 & 39.30 \\
\hline AVP & 5 & 8.19 & Ox-CBZ & 13 & 6.46 \\
\hline FB & 2 & 3.27 & LTG & 7 & 3.48 \\
\hline CNZ & 2 & 3.27 & LEV & 4 & 1.99 \\
\hline \multirow[t]{3}{*}{ PMD } & 1 & 1.63 & TOP & 3 & 1.49 \\
\hline & & & DFH & 2 & 0.99 \\
\hline & & & GPT & 1 & 0.99 \\
\hline Politerapia & 21 & 21 & Politerapia & 48 & 17.45 \\
\hline $\mathrm{DFH}+\mathrm{AVP}$ & 5 & 23.80 & $\mathrm{AVP}+\mathrm{CBZ}$ & 19 & 37.25 \\
\hline $\mathrm{DFH}+\mathrm{CBZ}$ & 4 & 19.04 & AVP + LTG & 9 & 17.64 \\
\hline $\mathrm{AVP}+\mathrm{CNZ}$ & 2 & 9.52 & CBZ + LEV & 5 & 9.80 \\
\hline $\mathrm{AVP}+\mathrm{CBZ}$ & 2 & 9.52 & $\mathrm{AVP}+\mathrm{DFH}$ & 4 & 7.84 \\
\hline $\mathrm{DFH}+\mathrm{CNZ}$ & 1 & 4.76 & $\mathrm{LTG}+\mathrm{OXC}$ & 2 & 3.92 \\
\hline $\mathrm{DFH}+\mathrm{FB}$ & 1 & 4.76 & $A V P+O X C$ & 2 & 3.92 \\
\hline $\mathrm{DFH}+\mathrm{PMD}$ & 1 & 4.76 & $\mathrm{CBZ}+\mathrm{DFH}$ & 1 & 1.96 \\
\hline $\mathrm{CBZ}+\mathrm{PMD}$ & 1 & 4.76 & $\mathrm{CBZ}+\mathrm{OXC}$ & 1 & 1.96 \\
\hline $\mathrm{CBZ}+\mathrm{CNZ}$ & 1 & 4.76 & $\mathrm{CBZ}+\mathrm{LTG}$ & 1 & 1.96 \\
\hline $\mathrm{CBZ}+\mathrm{DFH}+\mathrm{AVP}$ & 2 & 9.52 & $\mathrm{CBZ}+\mathrm{TOP}$ & 1 & 1.96 \\
\hline \multirow[t]{3}{*}{$\mathrm{CBZ}+\mathrm{FB}+\mathrm{AVP}$} & 1 & 4.76 & $\mathrm{LTG}+\mathrm{DFH}$ & 1 & 1.96 \\
\hline & & & $A V P+C B Z+L T G$ & 3 & 5.88 \\
\hline & & & $\mathrm{CBZ}+\mathrm{LTG}+\mathrm{LEV}$ & 2 & 3.92 \\
\hline Tratamiento no farmacológico & 18 & 18 & Tratamiento no farmacológico & 23 & 8.36 \\
\hline
\end{tabular}

CNZ: clonazepam; PMD: pirimidina. La administración de los medicamentos antiepilépticos en las mujeres embarazadas se divide en monoterapia o politerapia, aunque también hay mujeres que no reciben ningún tipo de tratamiento farmacológico. La cantidad de pacientes y los porcentajes se indican anteriormente, según cada estudio: el de 1996 y el de 2017

Los embarazos por cesárea aumentaron del 50 al $68 \%$, mientras que la proporción de uso de fórceps en el parto vaginal se redujo del 20 al $6 \%$. Con respecto a la indicación de cesárea, la desproporción cefalopélvica predominó en ambos estudios: $19 \%(n=11)$ en 1996 y 16\% ( $n=27)$ en 2017.

Los hijos de madres epilépticas mostraron características somáticas similares en ambas investigaciones, aunque el número de recién nacidos con bajo peso (hipotróficos) fue mayor en el estudio actual (del 3 al $18 \%)$, mientras que la proporción de fallecimientos neonatales fue similar (del 1\%) (Tabla 3). Una mayor proporción de niños expuestos al AVP fueron clasificados como hipotróficos al nacer (16 de estos 28 neonatos fueron expuestos a monoterapia, y 10 de 14 a politerapia) (Tabla 5). 
Tabla 3. Datos somatométricos neonatales

\begin{tabular}{|c|c|c|c|c|c|}
\hline \multicolumn{3}{|l|}{ Estudio de 1996} & \multicolumn{3}{|c|}{ Estudio de 2017} \\
\hline & $n=100$ & $\%$ & & $n=275$ & $\%$ \\
\hline Niñas & 59 & 59 & Niñas & 134 & 50 \\
\hline $\begin{array}{l}\text { Peso promedio } \\
\text { Mínimo } \\
\text { Máximo }\end{array}$ & $\begin{array}{l}3,081 \\
2,200 \\
4,000\end{array}$ & & $\begin{array}{c}\text { Peso promedio } \\
\text { Mínimo } \\
\text { Máximo }\end{array}$ & $\begin{array}{l}2,880 \\
1,355 \\
4,305\end{array}$ & \\
\hline $\begin{array}{l}\text { Altura promedio } \\
\text { Mínimo } \\
\text { Máximo }\end{array}$ & $\begin{array}{c}49.5 \\
44.5 \\
54\end{array}$ & & $\begin{array}{l}\text { Altura promedio } \\
\text { Mínimo } \\
\text { Máximo }\end{array}$ & $\begin{array}{l}48 \\
32 \\
54\end{array}$ & \\
\hline $\begin{array}{l}\text { CP promedio } \\
\text { Mínimo } \\
\text { Máximo }\end{array}$ & $\begin{array}{c}34.4 \\
31.5 \\
37\end{array}$ & & $\begin{array}{l}\text { CP promedio } \\
\text { Mínimo } \\
\text { Máximo }\end{array}$ & $\begin{array}{c}33.6 \\
28 \\
39\end{array}$ & \\
\hline Niños & 41 & 41 & Niños & 138 & 49 \\
\hline $\begin{array}{l}\text { Peso promedio } \\
\text { Mínimo } \\
\text { Máximo }\end{array}$ & $\begin{array}{l}3,081 \\
2,200 \\
4,000\end{array}$ & & $\begin{array}{c}\text { Peso promedio } \\
\text { Mínimo } \\
\text { Máximo }\end{array}$ & $\begin{array}{l}2,936 \\
1,010 \\
3,982\end{array}$ & \\
\hline $\begin{array}{l}\text { Altura promedio } \\
\text { Mínimo } \\
\text { Máximo }\end{array}$ & $\begin{array}{l}49.1 \\
45 \\
54\end{array}$ & & $\begin{array}{l}\text { Altura promedio } \\
\text { Mínimo } \\
\text { Máximo }\end{array}$ & $\begin{array}{c}48.5 \\
36.5 \\
53\end{array}$ & \\
\hline $\begin{array}{l}\text { CP promedio } \\
\text { Mínimo } \\
\text { Máximo }\end{array}$ & $\begin{array}{c}33.7 \\
29.5 \\
36\end{array}$ & & $\begin{array}{l}\text { CP promedio } \\
\text { Mínimo } \\
\text { Máximo }\end{array}$ & $\begin{array}{c}33.5 \\
17.7 \\
37\end{array}$ & \\
\hline Eutrófico & 83 & 83 & Eutrófico & 200 & 85.4 \\
\hline Hipertrófico & 13 & 13 & Hipertrófico & 8 & 2.9 \\
\hline Hipotrófico & 3 & 3 & Hipotrófico & 48 & 18 \\
\hline Abortos & 1 & 1 & Abortos & 3 & 1 \\
\hline
\end{tabular}

Cada estudio proporcionó datos somatométricos neonatales específicos sobre el peso, la altura y el perímetro cefálico, tanto de niños como de niñas. Las clasificaciones de peso específico neonatal y la información de los fallecimientos o abortos también se presentan en la tabla. Todas las medidas se muestran en número de casos y porcentajes.

La puntuación de Virginia Apgar a 1 y 5 min fue similar en ambos estudios. Los dismorfismos fueron más frecuentes en el estudio actual: la dismorfia menor se incrementó del 8 al 12\% y la mayor subió del 0 al 3\% (Tabla 4).

\section{Discusión}

Las mujeres embarazadas que asisten a la Clínica de Epilepsia del INPer son referidas principalmente por las instituciones de salud pública del país, que seleccionan el tratamiento antiepiléptico según los diagnósticos clínico-EEG. Con base a éstos, ajustamos la dosis de FAE favoreciendo la monoterapia (con excepción de la epilepsia generalizada resistente al tratamiento), y con ello buscamos proteger la salud materna e infantil y tratar las complicaciones perinatales de acuerdo con los estándares internacionales ${ }^{19-21}$.

Cabe destacar el aumento de la frecuencia de las crisis de inicio generalizado en el estudio actual (59.4\%), cantidad que se triplica en relación con la de hace 20 años. Este cambio epidemiológico en el tipo de epilepsia prevalente en los hospitales de referencia puede indicar una posible transición de un predominio causal de epilepsias sintomáticas vistas en diversos países en desarrollo ${ }^{22}$ a uno de epilepsias determinadas genéticamente observadas en naciones desarrolladas, motivadas por una posible reducción de causas que pueden prevenirse dentro de las medidas de salud pública, tales como las asociadas a infecciones prevenibles por vacunación (tuberculosis, meningitis y hemofilia), neurocisticercosis y 
Tabla 4. Malformaciones. Estudio de 2017.

\begin{tabular}{|c|c|c|}
\hline Dismorfismos por paciente & FAE & Dosis \\
\hline $\begin{array}{l}\text { Defectos del tubo neural } \\
\text { Riñón poliquístico } \\
\text { Hipospadias } \\
\text { Síndrome de Arnold Chari de tipo } 2\end{array}$ & $\begin{array}{l}\text { AVP } \\
\text { LTG }\end{array}$ & $\begin{array}{c}1,200 \\
225\end{array}$ \\
\hline Defectos del tubo neural & CBZ & 600 \\
\hline $\begin{array}{l}\text { Hidrocele bilateral } \\
\text { Criptorquidia derecha }\end{array}$ & CBZ & 300 \\
\hline $\begin{array}{l}\text { Hidrocele bilateral } \\
\text { Apéndice preauricular izquierdo }\end{array}$ & CBZ & 600 \\
\hline $\begin{array}{l}\text { Hidrocefalia } \\
\text { Micropene } \\
\text { Asimetría craneal: aplastamiento temporal izquierdo } \\
\text { Polidactilia bilateral (en ambas manos) } \\
\text { Tibia vara en la pierna derecha }\end{array}$ & CBZ & 400 \\
\hline $\begin{array}{l}\text { Malformaciones congénitas de la cara y el cuello } \\
\text { Malformación congénita de la nariz } \\
\text { Malformación congénita de las cámaras cardíacas y de sus conexiones } \\
\text { Vena genital anormal } \\
\text { Dos arterias genitales anormales }\end{array}$ & $\begin{array}{l}\text { AVP } \\
\text { CBZ } \\
\text { LTG }\end{array}$ & $\begin{array}{c}1,200 \\
600 \\
50\end{array}$ \\
\hline $\begin{array}{l}\text { Microcefalia, braquicefalia } \\
\text { Teletelia } \\
\text { Pezones invertidos }\end{array}$ & $\begin{array}{l}\text { AVP } \\
\text { LTG }\end{array}$ & $\begin{array}{c}1,600 \\
225\end{array}$ \\
\hline Displasia de cadera & $\begin{array}{l}\text { AVP } \\
\text { LTG } \\
\text { TOP }\end{array}$ & $\begin{array}{c}800 \\
100 \\
25\end{array}$ \\
\hline $\begin{array}{l}\text { Displasia de cadera } \\
\text { Hipotelorismo } \\
\text { Estrechamiento bitemporal } \\
\text { Sutura metópica frontal }\end{array}$ & AVP & 600 \\
\hline $\begin{array}{l}\text { Pie izquierdo equino } \\
\text { Osteocromos en ambos pies } \\
\text { Pie equino varo }\end{array}$ & $\begin{array}{l}\text { AVP } \\
\text { CBZ } \\
\text { CBZ }\end{array}$ & $\begin{array}{c}750 \\
1,200 \\
1,600\end{array}$ \\
\hline Apéndice preauricular izquierdo & CBZ & 300 \\
\hline Mancha mongólica & $\begin{array}{l}\text { AVP } \\
\text { AVP } \\
\text { AVP } \\
\text { AVP } \\
\text { AVP } \\
\text { AVP } \\
\text { AVP } \\
\text { AVP + LTG } \\
\text { AVP + CBZ } \\
\text { CBZ } \\
\text { CBZ } \\
\text { CBZ } \\
\text { LTG } \\
\text { LTG } \\
\text { Sin tratamiento y con mancha mongólica }\end{array}$ & $\begin{array}{c}1,200 \\
1,200 \\
1,000 \\
750 \\
600 \\
400 \\
1,200 \\
800+200 \\
800+400 \\
1,600 \\
400 \\
40 \\
100 \\
200 \\
5 \text { mujeres }\end{array}$ \\
\hline
\end{tabular}

En referencia a cada paciente del estudio de 2017, se muestra el tipo de dismorfia, el tipo de medicación antiepiléptica utilizada y su dosis 
lesiones en la cabeza asociadas con accidentes automovilísticos, entre otras ${ }^{23-26}$.

La prevalencia de la epilepsia con convulsiones generalizadas previas al embarazo explica la alta frecuencia de pacientes que mantuvieron el uso del AVP durante el embarazo (45\%). Se debe reconsiderar el alto consumo de AVP en mujeres jóvenes con epilepsia con convulsiones generalizadas, ya que aumenta el riesgo de teratogénesis en caso de que estén embarazadas ${ }^{10,11,19,20}$. Es esencial llevar a cabo campañas entre los médicos de las instituciones públicas que promuevan la reducción del consumo del AVP y que favorezcan el uso del FAE, que tiene una alta eficiencia y un amplio espectro (motor y no motor: tónico-clónico, mioclónico y ausente), pero con una menor capacidad de teratotoxicidad ${ }^{27-29}$. Conviene especificar que las instituciones obstétricas donde recibimos a estas pacientes embarazadas pueden hacer poco al respecto cuando ingresan después de la semana 20 de gestación $n^{10,19}$.

El número de pacientes que descontinuaron el FAE de forma transitoria cuando se quedaron embarazadas se redujo del 50 al $24 \%$, lo que pudo significar un mejor conocimiento de la enfermedad y una buena recomendación de su médico. Sin embargo, esta reducción no se reflejó en el número de complicaciones perinatales, pues éstas se mantuvieron similares en ambos estudios, lo que sugiere un origen independiente al de las complicaciones obstétricas ${ }^{30}$, por lo que surge la necesidad de estudiar otros factores potenciales como la vigilancia obstétrica, la nutrición y la genética.

La frecuencia de cesáreas se mantuvo elevada (entre el 50 y $68 \%$ ) y el uso de fórceps se redujo significativamente (del 20 al 6\%), lo que puede reflejar el miedo de los obstetras por las posibles complicaciones durante el parto ${ }^{31}$, lo que obliga a instituir ciertas recomendaciones para promover el parto vaginal en pacientes embarazadas que sufren de epilepsia. La cesárea es más común en la población de alto riesgo, pero sigue siendo frecuente en los embarazos normales, y se estima que uno de cada tres embarazos será por cesárea ${ }^{32}$. Las investigaciones futuras deberían centrarse en un análisis más profundo con respecto al aumento y mantenimiento de este tipo de intervención quirúrgica a lo largo del tiempo, incluso si el embarazo no presenta ningún riesgo.

Los datos somatométricos son similares en los recién nacidos masculinos y femeninos, pero se observa una diferencia importante en los lactantes varones lo cual corresponde a la literatura en la cual se ha visto que, en general, éstos suelen tener valores más elevados de peso y circunferencia cefálica ${ }^{33}$.
En proporción al mayor uso del AVP durante el embarazo, hubo un aumento del 8 al $45 \%$ entre el estudio de 1996 y el de 2015; asimismo, se observó una mayor frecuencia de hipotrofia en niños expuestos, del 3 al $18 \%$, respectivamente.

Aunque frecuentemente se ha asociado el AVP con malformaciones importantes ${ }^{34,35}$, no existen reportes de su relación con un bajo peso al nacer, lo que puede representar características genéticas o epigenéticas particulares de nuestra población. Otra diferencia local es la mayor frecuencia del uso de AVP de magnesio en comparación con América del Norte y Europa, donde no está permitido, lo que podría representar una línea de investigación futura para determinar el impacto en los efectos perinatales de agregar magnesio a la fórmula del AVP ${ }^{36,37}$.

En el estudio actual se duplicó la frecuencia de los dismorfismos menores (del 8 al 12\%) y de los mayores (del 0 al $3 \%$ ), en una proporción similar a la del aumento del uso de AVP durante el embarazo, que pasó del 8 al 45\%. En 15 de 33 niños expuestos con alguna malformación se utilizó este fármaco, y 3 de 7 niños con malformaciones importantes fueron expuestos a la politerapia de AVP. Estos resultados no excluyen el efecto teratogénico de otros antiepilépticos (la CBZ se asoció con el $33 \%$ de los niños malformados), pero muestra un incremento proporcional de malformaciones asociadas al aumento del uso de AVP, especialmente en dosis altas ${ }^{6,11}$ en la población mexicana.

En esta investigación se presentaron importantes limitaciones que deben ser mencionadas. Debido a que los pacientes fueron estudiados de forma retrospectiva, se dio la necesidad de excluir diversos casos teratogénicos observados cuando la información era inconsistente y su seguimiento imposible, por lo que no se pudo llevar a cabo una evaluación cognitiva ni del desarrollo neurológico.

Estos resultados demuestran la necesidad de futuros estudios prospectivos que definan las peculiaridades genéticas de la población mexicana, factor que podría estar involucrado en el resultado perinatal. Asimismo, se necesita más investigación según el tipo de AVP que se administra durante el embarazo, ya sea de sodio o de magnesio, lo que demuestra un posible efecto teratogénico mayor que nunca se ha analizado en la población mexicana. También es importante que se realicen estudios acerca de la teratogenicidad y de un mayor desarrollo neurológico, para que los servicios de salud mexicanos creen campañas nacionales que disuadan los riesgos del uso del AVP en mujeres fértiles. 
Tabla 5. Hipotrofia y FAE. Estudio de 2017

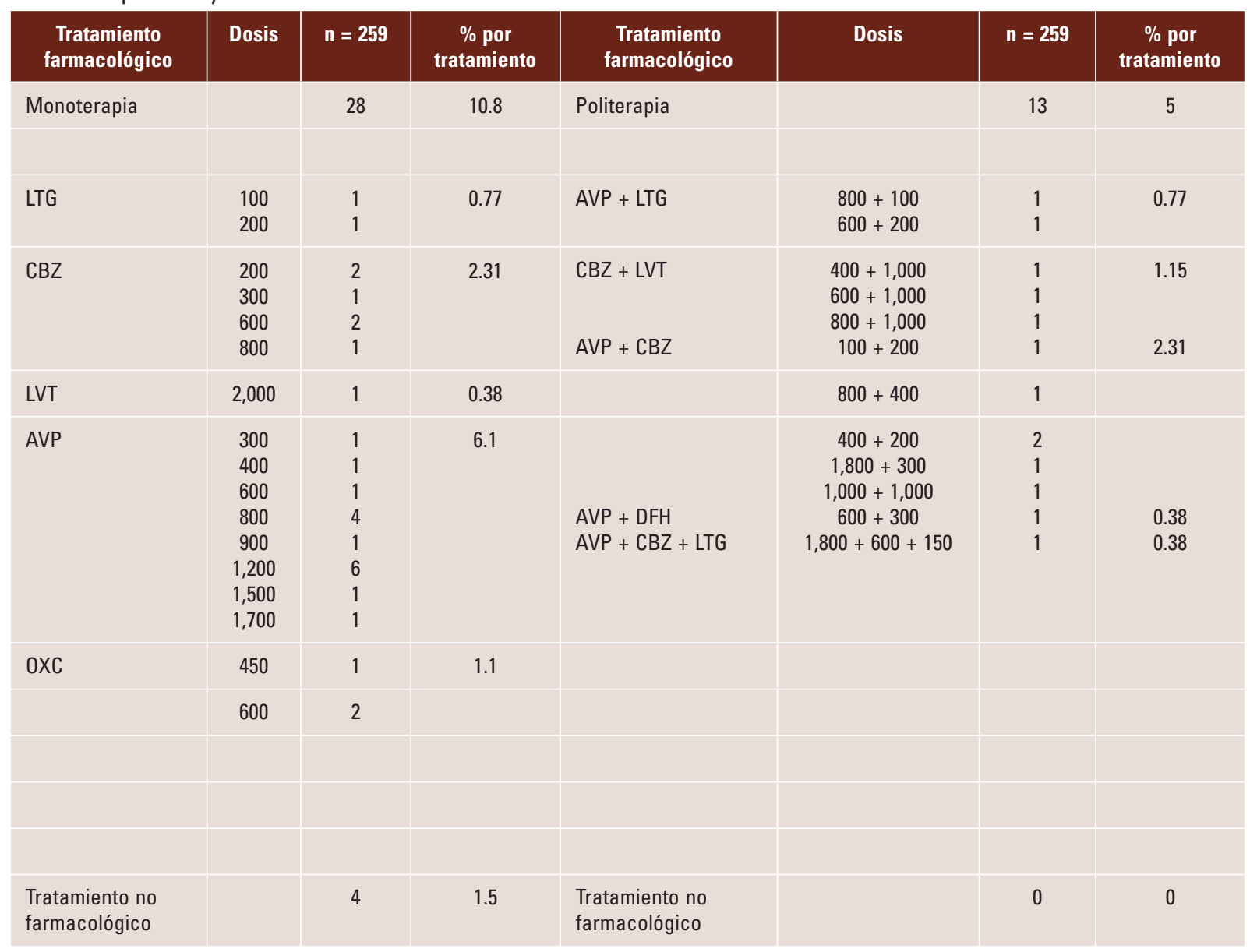

Datos referentes a cada lactante hipotrófico del estudio de 2017, el tipo de medicación antiepiléptica utilizada y su dosis. También se muestran los casos sin tratamiento farmacológico

\section{Conclusiones}

Los hallazgos previos deben alertar a las instituciones públicas sobre los efectos teratogénicos específicos del AVP y buscar otras estrategias para el tratamiento antiepiléptico dirigido a las mujeres con epilepsia en edad reproductiva, debido a la evidencia con respecto a un aumento en malformaciones menores y mayores, así como a datos de hipotrofia. En espera de que se proporcione educación y prevención para futuros embarazos, se recomienda un cambio en los FAE. Se busca implementar en todas las instituciones de salud tratamientos alternativos de FAE de nueva generación, para ofrecerlos a las mujeres antes de que se embaracen. Además de los resultados de este artículo, hay datos suficientes alrededor del mundo que indican un tratamiento para la epilepsia generalizada basado en la evidencia. Estos hallazgos no sólo son relevantes para las mujeres embarazadas, sino que surge la cuestión acerca de su uso seguro en toda la población de mujeres jóvenes.

\section{Conflictos de interés}

No existen conflictos de interés para publicar este material.

\section{Fuente de financiamiento}

El siguiente trabajo ha sido financiado por el Instituto Nacional de Perinatología Isidro Espinosa de los Reyes.

\section{Agradecimientos}

Por sus valiosas contribuciones al presente trabajo, nuestros más sinceros agradecimientos a la Dra. Rocío Ivonne de la Vega Morales y al Dr. Jorge Armando Barriguete Meléndez. 


\section{Bibliografía}

1. Katz O, Levy A, Wiznitzer A, Sheiner E. Embarazo y resultados perinatales en mujeres epilépticas: un estudio poblacional. J Matern Fetal Neonatal Med. 2006;19(1):21-5.

2. Borthen I, Eide M, Veiby G, Daltveit A, Gilhus N. Complicaciones durante el embarazo en mujeres con epilepsia: un estudio de cohorte poblacional. BJOG. 2009;116(13):1736-42.

3. Anuario Estadístico 2016. Instituto Nacional de Perinatología Isidro Espinosa de los Reyes. Ciudad de México, México; 2017.

4. Thomas SV, Syam U, Devi JS. Indicadores de convulsiones en mujeres con epilepsia durante el embarazo. Epilepsia. 2012;53(5):85-8

5. Cantwell R, Clutton-Brock T, Cooper G, Dawson A, Drife J, Garrod D et al. Salvando las vidas de las madres: un análisis de las muertes maternas para hacer más segura la maternidad: 2006-2008. El octavo informe de las consultas confidenciales acerca de las muertes maternas en el Reino Unido. BJOG. 2011;118 (Suppl 1):1-203.

6. Tomson T, Battino D, Bonizzoni E. EURAP Study Group. Riesgo dosis dependiente de malformaciones con fármacos antiepilépticos: un análisis de los datos del registro de epilepsia y embarazo del EURAP. Lancet Neurol. 2011;10(7):609-17

7. Artama M, Auvinen A, Raudaskoski T, Isojarvi I, Isojarvi J. Uso de fármacos antiepilépticos en mujeres con epilepsia y malformaciones congénitas en su descendencia. Neurology. 2005;64:1874-8

8. Borthen I, Eide MG, Daltveit AK, Gilhus NE. Resultado obstétrico en mujeres con epilepsia: un estudio retrospectivo basado en el hospital. BJOG. 2011;118(8):956-65.

9. Mølgaard-Nielsen D, Hviid A. Los medicamentos antiepilépticos de nueva generación y el riesgo de defectos de nacimiento importantes. JAMA 2011;305(19):1996-2002.

10. Tomson T, Marson A, Boon P. El valproato en el tratamiento de la epilepsia en niñas y mujeres en edad fértil. Epilepsia. 2015;56(7):1006-19.

11. Morrow J, Russell A, Guthrie E, Parsons L, Robertson I, Waddell R, et al. Riesgos de malformación de los fármacos antiepilépticos en el embarazo: un estudio prospectivo del registro de epilepsia y embarazo del Reino Unido. J Neurol Neurosurg Psychiatry. 20016;77(2):193-8.

12. Garza-Morales S, Ibarra-Puig JM, Poblano-Luna A, Mayén-Molina DG, Córdova-López S. Epilepsia y embarazo. Estudio prospectivo de 100 casos. Ginecología y obstetricia de México. 1996:64:449-54.

13. Ibarra-Puig J, Córdova-López S, Fernández M. Epilepsia y embarazo. Rev. Neurología, Neurocirugía y Psiquiatría. 1992;32:59-68.

14. Poblano A, Belmont A, Sosa J. Los efectos de la exposición prenatal a la carbamazepina sobre los potenciales evocados auditivos del tronco cerebral en bebés de madres epilépticas. J Child Neurol. 2002;17(5):364-8.

15. Fisher RS, van Emde Boas W, Blume W, Elger C, Genton P, Lee P, et al. Convulsiones epilépticas y epilepsia: Definiciones propuestas por la Liga Internacional contra la Epilepsia (ILAE) y por el Buró Internacional para la Epilepsia (IBE). Epilepsia. 2005;46(4):470-2.

16. Norma Oficial Mexicana NOM-007-SSA2-1993, para la atención de la mujer durante el embarazo, parto y puerperio y del recién nacido. Criterios y procedimientos para la prestación del servicio.

17. Guía de práctica clínica. Diagnóstico y tratamiento oportuno del estado epiléptico en el primer y segundo nivel de atención: Guía de referencia rápida. Gobierno Federal: Consejo de salubridad general. Catálogo de guías de práctica clínica: S5A-092-08.

18. Vigilancia Europea de Anomalías Congénitas. Guía 1.3 EUROCAT y documentos de referencia: instrucciones para el registro y vigilancia de anomalías congénitas. Disponible en: http://www.eurocatnetwork.eu/content/EUROCAT-Guide-1.3

19. Harden CL, Hopp J, Ting TY, Pennell PB. Cuestiones administrativas para mujeres con epilepsia: enfoque en el embarazo (un análisis basado en la evidencia): I. Complicaciones obstétricas y cambio en la frecuencia de las crisis: Informe del subcomité de estándares de calidad y del subcomité de evaluación de terapéutica y tecnología de la Academia
Americana de Neurología y de la Sociedad Americana de Epilepsia. Epilepsia. 2009;50(5):1229-36.

20. Harden CL, Meador KJ, Pennell PB. Cuestiones administrativas para mujeres con epilepsia: enfoque en el embarazo (un análisis basado en la evidencia): II. Teratogénesis y resultados perinatales: Informe del subcomité de estándares de calidad y del subcomité de evaluación de terapéutica y tecnología de la Academia Americana de Neurología y de la Sociedad Americana de Epilepsia. Epilepsia. 2009;50(5):1237-46.

21. Harden CL, Pennell PB, Koppel BS. Cuestiones administrativas para mujeres con epilepsia: enfoque en el embarazo (un análisis basado en la evidencia): III. Vitamina K, ácido fólico, niveles sanguíneos y lactancia materna: Informe del subcomité de estándares de calidad y del subcomité de evaluación de terapéutica y tecnología de la Academia Americana de Neurología y de la Sociedad Americana de Epilepsia. Epilepsia. 2009;50(5):1247-55.

22. Hauser WA. Epidemiología de la epilepsia. World Neurology. 2000; 15(1):6-8.

23. Forsgren L, Hesdorffer D. Epidemiología y pronóstico de la epilepsia en el tratamiento de la epilepsia, $3 .^{a}$ ed. Shorvon S, Perucca E, Engel J, editores. Blackwell Publishing Ltd.; 2009. p. 21-32.

24. Bharucha NE, Shorvon SD. Epidemiología en países en desarrollo. En Engel Jr J, Pedley TA, editores. Epilepsy: A comprehensive textbook. Philadelphia: Lippincott Raven Press; 1997. p. 105-18.

25. Acevedo C, OMS, ILAE e IBE. Informe sobre la epilepsia en Latinoamérica. Panamá; 2008

26. Camfield C, Camfield P. Causas prevenibles e inevitables de la epilepsia de inicio en la infancia con retraso mental. Pediatrics. 2007;120(1):52-5.

27. Morrow J, Russell A, Guthrie E. Riesgos de malformación de los fármacos antiepilépticos en el embarazo: un estudio prospectivo del Registro de epilepsia y embarazo en el Reino Unido. J Neurol Neurosurg Psychiatry. 2006;77:193-8

28. Yamamoto $T$, Yamazoe $T$, Fujimoto $A$. La efectividad y el papel de la lamotrigina en el cambio de paradigma hacia nuevos fármacos antiepilépticos: una estrategia terapéutica propuesta después de una investigación de un gran número de casos en un solo hospital. Brain Nerve. 2014;66(1):59-69.

29. Diav-Citrin O, Shechtman S, Zvi N, Finkel-Pekarsky V, Ornoy A. ¿Es seguro el uso de la lamotrigina durante el embarazo? Un estudio observacional, prospectivo y comparativo. Birth Defects Res. 2017; 109(15):1196-203

30. Richmond JR, Krishnamoorthy $P$, Andermann E. Epilepsia y embarazo: una perspectiva obstétrica. Am J Obstet Ginecol. 2004:191(6):2174-5.

31. Borthen I, Eide MG, Daltveit AK. Resultado del parto en mujeres con epilepsia: un estudio de cohorte poblacional. BJOG. 2010;117:1537-43.

32. Weiss AJ, Elixhauser A, Andrews RM. Características de los procedimientos de las salas de operación en los hospitales de los Estados Unidos, 2011. HCUP Statistical Brief \#170. Rockville, MD, EE. UU.: Agencia para la investigación y la calidad de la atención de la salud; 2014. [Recuperado el 24 de septiembre de 2015]. Disponible en: http:// www.hcup-us.ahrq.gov/reports/statbriefs/sb170-Operating-Room-Procedures-United-States-2011.pdf

33. Kurtoglu S, Hatipoglu N, Mazicioglu MM, Akin MA, Çoban D, Gökoglu S et al. Peso corporal, longitud y circunferencia de la cabeza al nacer en un grupo de recién nacidos turcos. J Clin Res Pediatr Endocrinol. 2012;4(3):132-9

34. Veiby G, Daltveit AK, Engelsen BA, Gilhus NE. Restricción del crecimiento fetal y defectos de nacimiento con fármacos antiepilépticos nuevos y antiguos durante el embarazo. J Neurol. 2014;261(3):579-88

35. DiLiberti JH, Farndon PA, Dennis NR, Curry CJ. El síndrome del valproato fetal. Am J Med Genet. 1984;19(3):473-81.

36. Bogden JD, Thind IS, Louria DB, Caterini H. Concentraciones de metal en la sangre materna y en la del cordón umbilical, y bajo peso al nacer: un estudio de casos y controles. Am J Clin Nutr. 1978;31(7):1181-7.

37. Tsang RC, Oh W. Niveles séricos de magnesio en lactantes de bajo peso al nacer. Am J Dis Child. 1970;120(1):44-8. 